\title{
La didattica dell'italiano a distanza: un case study
}

Paolo Matteucci

Dalhousie University matteucci@dal.ca

\begin{abstract}
Questo saggio si propone il fine di misurare l'impatto della transizione dalla didattica in aula a quella a distanza nel contesto dei corsi di italiano per principianti offerti, nel semestre autunnale 2020, dal Programma di Italiano dell'università canadese Dalhousie. Prendendo simultaneamente in considerazione i risultati del feedback formale e informale fornito dagli allievi, i dati raccolti tramite la ricerca-azione e l'osservazione dei corsi da parte di specialisti esterni, e una molteplicità di parametri valutativi, il presente intervento mira a delineare alcuni vantaggi e svantaggi dell'insegnamento a distanza dell'italiano come lingua straniera.
\end{abstract}

Parole-chiave: Didattica dell'italiano. Glottotecnologie. Ricerca-azione.

RESUMO: Este artigo analisa o impacto da transição das aulas presenciais ao formato virtual, especificamente no contexto das três seções do curso "Italiano para Iniciantes" oferecido no segundo semestre de 2020 pelo Programa de Italiano da Universidade de Dalhousie, Canadá. Justapondo os feedbacks formais e informais dos alunos, os dados recebidos da observação de terceiros através de pesquisa-ação e as avaliações de diversos indicadores de sucesso, este artigo visa delinear algumas vantagens, bem como os principais desafios, do ensino a distância do italiano enquanto idioma estrangeiro.

Palavras-chave: Didática do italiano. Glototecnologias. Pesquisa-ação.

ABSTRACT: This paper scrutinizes the impact of the transition from faceto-face teaching to online instruction, in the context of the Italian for Beginners courses offered in the fall semester 2020 by the Italian Program at Dalhousie University, Canada. By juxtaposing the student's formal and informal feedback, the input received from third-party observation via actionresearch, and the assessment of a variety of indicators of success, this paper aims at outlining some advantages, as well as the main challenges, inscribed into at-distance teaching of Italian as a foreign language.

Key words: Teaching Italian as a foreign language. At-distance instruction. Action-research. 


\section{Introduzione}

$\mathcal{N}$ el contesto di emergenza sanitaria sviluppatasi, su scala mondiale, nel corso dell'anno 2020, la pratica dell'insegnamento dell'italiano per via telematica ha registrato un significativo incremento quantitativo e qualitativo che ha interessato, in vare parti del globo, un grande numero di istituzioni formative di diverso ordine e grado. A distanza di alcuni mesi dalla messa in atto di alcune misure di profilassi sanitaria quali la chiusura quasi totale dei campus e delle aule di molte università italiane e internazionali, il presente saggio mira a tracciare un bilancio degli effetti apportati dalla transizione da una pratica d'insegnamento svolta di persona a una modalità didattica interamente effettuata a distanza nel contesto dei corsi di lingua italiana offerti nel semestre autunnale dell'anno accademico 2020/21 dal Programma di Italiano dell'Università Dalhousie, in Canada.

A partire dal case study oggetto di discussione, questo intervento propone di mettere a confronto le metodologie di insegnamento-apprendimento adottate in aula ed online, di misurare gli effetti della didattica a distanza sulle strategie e sulle pratiche adottate a lezione e, soprattutto, di enucleare alcune specificità tipiche dell'insegnamento della lingua italiana a distanza a partire dalla valutazione e dalla autovalutazione del processo di insegnamentoapprendimento dal punto di vista sia del personale docente sia degli studenti.

Nell'identificare una serie di caratteristiche e di problematiche specificamente appartenenti ai corsi di italiano cui è dedicata, la presente indagine mira a fornire una serie di indicazioni di ordine generale e a delineare una possibile serie di strumenti euristici e di potenziali punti di partenza per un ulteriore e più approfondito dibattito sui benefici e sulle difficoltà pertinenti all'insegnamento della lingua italiana, nel contesto dell'istruzione superiore, per via telematica. 


\section{Metodologia e strumenti}

Nel quadro teorico sviluppato da John Biggs (1996, 2003 e 2012), la valutazione del successo di un processo di insegnamento non può essere disgiunta da una considerazione olistica della didattica quale sistema complesso di relazioni intercorrenti tra allievi, docenti, contenuti, attività di classe, pratiche valutative e apprendimento nel suo insieme. In un'ottica epistemologica prettamente costruttivista, infatti, per Biggs il successo di un determinato percorso formativo è misurabile nel raggiungimento, o meno, di un allineamento ("alignment") tra processo cognitivo, pratica didattica e raggiungimento degli obiettivi formativi.

Al fine di misurare tale allineamento in un contesto specifico, ovvero i corsi di lingua italiana dell'autunno 2020 l'Università Dalhousie, il presente studio si avvale della misurazione sistematica e periodica di alcuni indicatori che vertono, principalmente, sulla percezione del successo didattico da parte degli allievi. Tale misurazione, ai fini del presente studio, è stata effettuata attraverso il prelievo e la comparazione di dati statistici raccolti a intervalli regolari nel tempo; la periodica somministrazione agli allievi dei corsi di italiano di questionari e sondaggi e la valutazione del conseguimento, da parte degli allievi, delle capacità comunicative che costituiscono il fondamento di un corso di italiano come lingua straniera a livello universitario.

Sulla falsariga del lavoro sviluppato nei primi anni '90 da Ramsden (1991), una molteplicità di studi ha nel corso del tempo evidenziato l'importanza insita nel costante monitoraggio della percezione, da parte degli studenti, dei risultati ottenuti nelle diverse fasi del processo di apprendimentoinsegnamento. La raccolta e lo scrutinio del feedback degli allievi costituiscono in particolare, come è stato suggerito tra gli altri da Wiliam (2011), uno strumento efficace per la valutazione e l'autovalutazione delle traiettorie sviluppate da tutti i partecipanti al percorso didattico. Per le suddette ragioni, 
il presente studio è largamente basato sull'input valutativo ed autovalutativo fornito, nel corso del semestre, dagli iscritti ai corsi. Tale input, è importante sottolineare in chiave preliminare, è stato raccolto in maniera distaccata ed indipendente dalla misurazione aritmetica del successo degli studenti (esprimibile, ad esempio, attraverso i voti conseguiti dagli allievi), in ossequio alle indicazioni fornite dai diversi studi_tra cui, in particolare, Black \& Wiliam (1998) e Gibbs \& Simpson (2004)—che hanno sottolineato l'importanza di separare chiaramente, al momento della somministrazione e della interpretazione del feedback degli allievi, la sfera cognitiva e partecipativa da quella docimologica.

Per la presente ricerca sono stati utilizzati:

- dati statistici e quantitativi relativi al numero di iscritti ai corsi, al tasso di successo degli studenti ed al numero percentuale di abbandoni;

- feedback degli studenti raccolto tramite sondaggi a risposta semplice, a risposta aperta, a riposta affermativa/negativa e a risposta per parole-chiave;

- feedback degli studenti raccolto tramite questionari formativi (in progress) e sommativi (finali), contenenti domande a risposta multipla, domande a risposta aperta e quesiti di classificazione di concetti, idee e valori per ordine di importanza;

- feedback informale raccolto a voce (in classe e nelle ore di ricevimento) e per via epistolare;

- moduli di autovalutazione dell'operato del personale docente;

- feedback, osservazioni e commenti da parte di osservatori esterni;

- raccolta ed elaborazione di informazioni e di dati emersi nel corso di gruppi di lavoro, riunioni e incontri a tema con i colleghi.

\section{Triangolazione e ricerca-azione}


Nell'ambito della glottodidattica moderna (Pozzo 1993 e 2003; Tripp 2005) la ricerca-azione costituisce una efficace pratica di indagine conoscitiva finalizzata all'affinamento delle strategie e delle metologie didattiche adottate all'interno di una specifica classe o comunità di apprendimento. Più specificatamente, nella ricerca-azione il feedback che viene scambiato tra docenti e allievi viene messo a confronto con i risultati dell'osservazione delle dinamiche di classe da parte di osservatori esterni che, in un vero e proprio percorso di triangolazione di prospettive e di punti di vista, presenziano alle lezioni al fine di misurare la riuscita degli obiettivi didattici e formativi stabiliti, prima dell'osservazione dei corsi, con il docente.

Nel contesto della presente indagine, ai corsi di lingua italiana è stata invitata in qualità di osservatrice esterna la dott.ssa Kate Crane, specialista di teorie e pratiche pedagogiche del Dalhousie University Centre for Learning and Teaching (lt @dal.ca). La sua partecipazione alle lezioni si è svolta su base volontaria, su invito del docente e, per motivi logistici, soltanto in un numero limitato di occasioni. La collaborazione con la dott.ssa Crane si è articolata in tre fasi principali, rispettivamente consistenti nella discussione preventiva delle problematiche e delle difficoltà organizzative legate all'utilizzo della piattaforma online adottata per i corsi di italiano, nell'osservazione strutturata delle dinamiche di classe nel corso delle lezioni e nella rielaborazione, a posteriori, delle informazioni raccolte nel corso del monitoraggio sia delle lezioni che dei materiali didattici messi a disposizione degli studenti.

Al termine del nostro breve percorso di ricerca-azione sono state raggiunte alcune conclusioni di ordine specifico, principalmente legate all'utilizzo delle risorse tecnologiche, e alcune considerazioni di ordine generale, principalmente vertenti sull'importanza di una accorta lettura dei tempi di partizione delle lezioni e sulle potenzialità insite in uno stile didattico 
incentrato su modalità accattivanti e stimolanti di presentazione, agli studenti, del materiale multimediale.

Dall'esperienza di triangolazione effettuata nei nostri corsi di lingua italiana è inoltre emerso che, per quanto riguarda la ricerca-azione, nell'ambito della didattica a distanza il tradizionale utilizzo di supporti tecnici come registratori vocali e apparecchiatura fotografica viene reso obsoleto dalla strumentazione attualmente disponibile. Il fatto che, per via telematica, l'osservazione possa essere sviluppata in maniera remota e invisibile sembra superare i limiti di ogni convenzionale distinzione tra osservazione emica ed osservazione etica (Harris 1983). Nel contesto della didattica a distanza, inoltre, l'invisibilità dell'osservatore esterno permette di eludere alcuni importanti paradossi dell'osservazione che si verificano nella ricerca-azione tradizionale (Bruschi \& Matteucci 2003).

\section{Dialogo e autoaggiornamento}

Nel corso delle conversazioni formali e informali tra colleghi, così come nel contesto di molteplici riunioni e gruppi di lavoro, sono emersi alcuni comuni punti di interesse, relativi all'insegnamento per via telematica, che si dipanano in maniera trasversale tra il corpo docente, indipendentemente dal tipo e dal livello dei corsi offerti e dal loro ambito disciplinare. Tra i punti focali emersi con maggiore frequenza nelle discussioni collegiali si annoverano:

- l'inopportunità e l'impossibilità, in un contesto telematico, di riprodurre pedissequamente il contenuto e le pratiche d'insegnamento adottate di persona;

- l'urgenza per ogni docente di intraprendere percorsi, individuali e collaborativi, di continuo aggiornamento ed autoformazione; 
- la necessità di sviluppare una attenta riflessione relativa alla scelta e all'uso delle piattaforme telematiche utilizzate per l'insegnamento online;

- l'importanza di architettare una efficace e accorta organizzazione dei materiali didattici messi a disposizione degli studenti sui siti internet dei corsi;

- l'inderogabile necessità di sviluppare pratiche docimologiche, di tipo sia formativo che sommativo, appropriate per un contesto di apprendimento svolto esclusivamente a distanza;

- l'opportunità di sviluppare pratiche valutative obiettive, e trasparenti, rispondenti ai diversi stili di apprendimento degli allievi, in un contesto didattico radicalmente diverso da quello che si sviluppa in aula.

\section{Sincronicità e asincronicità: verso un formato ibrido}

Le modalità di realizzazione di un corso universitario a distanza possono essere suddivise in due categorie principali. Nella modalità asincrona, ogni studente accede individualmente ai materiali che sono disponibili in linea e che vengono aggiornati, di solito, su base settimanale o infrasettimanale. Nella cornice asincrona, le lezioni non si svolgono mai nella forma di riunione di classe plenaria. Ciascun allievo completa individualmente le attività disponibili disponibili sul sito del corso, studia separatamente dagli altri studenti e completa autonomamente gli esercizi e i compiti.

Nel formato sincrono, invece, un corso si sviluppa attraverso uno o più incontri plenari settimanali a cui tutti gli studenti devono partecipare, così come avverrebe per le lezioni in aula. Nell'intervallo temporale compreso tra una sessione sincrona e la successiva, ciascun allievo consulta autonomamente i materiali affissi sul sito del corso e completa in maniera asincrona le attività, gli esercizi e i compiti disponibili in linea.

Ciascuno dei due formati presenta notevoli vantaggi ed evidenti svantaggi. Nel semestre autunnale 2020, a Dalhousie sono stati offerti corsi di 
italiano a distanza di formato ibrido, comprendenti un incontro plenario sincrono (online) settimanale, di 80 minuti e con frequenza e partecipazione obbligatorie, e una lezione asincrona, anch'essa settimanale, il cui completamento comporta l'impiego di circa ulteriori 80 minuti. A queste due scadenze settimanali vanno aggiunti i compiti e le prove di valutazione, il cui completamento può essere effettuato in qualsiasi momento della settimana purché antecedentemente all'inizio della lezione sincrona plenaria.

Dal feedback degli studenti è emerso che:

- la stragrande maggioranza degli studenti preferirebbe, se possibile, svolgere le lezioni di persona e in aula;

- più del 60\% degli studenti trova il formato ibrido produttivo, in quanto la componente sincrona permette di mantenere un contatto diretto con gli altri allievi e con il docente ed agevola l'organizzazione e la pianificazione settimanale dell'apprendimento, mentre la componente asincrona permette di rielaborare i contenuti della sessione sincrona in maniera autonoma e senza stringenti vincoli temporali;

- circa il 10\% degli allievi preferisce il formato completamente asincrono, spesso per motivi di impegni personali, familiari o lavorativi;

- quasi il 20\% degli studenti non ha preferenze in merito alla suddivisione del corso in componenti sincrone o asincrone.

\subsection{Componenti sincrone}

Le componenti sincrone dei corsi di italiano contribuiscono, secondo il feedback degli studenti, a rafforzare il senso di appartenenza alla classe, a cadenzare il ritmo dell'apprendimento con andamento settimanale e infrasettimanale, a stabilire un punto di contatto e di socialità tra i membri del corso ed a valorizzare le pratiche di apprendimento centrate sull'interazione, sull'aiuto reciproco e sulla collaborazione. Alcuni allievi sottolineano in 
particolare l'importanza di poter, nelle sessioni sincrone, dialogare e porre domande ai compagni ed al docente, l'importanza di ricevere tempestivamente assistenza e aiuto nel corso delle attività di classe (siano esse plenarie, a gruppi o a coppie) e sottolinea l'impossibilità di interagire proficuamente con i propri pari in un contesto puramente asincrono.

Dal punto di vista del docente, è emerso che nella pianificazione e nella realizzazione delle attività sincrone è importante prendere accortamente in considerazione i limiti tecnici e strutturali della piattaforma telematica che viene utilizzata. Molte delle principali piattaforme di collaborazione in linea attualmente disponibili, infatti, limitano la quantità e la qualità della condivisione di dati in tempo reale, spesso per motivi legati al design della piattaforma stessa oppure per ragioni di sicurezza e vincoli di privacy (nel nostro caso, stabiliti trasversalmente dall'Università per tutti i suoi corsi).

Alcuni importanti fattori tecnici da prendere attentamente in considerazione sono inoltte il tipo di lavagna elettronica offerto dalla piattaforma utilizzata, la gamma di funzionalità effettivamente disponibili per la condivisione di materiali multimediali, la possibilità di suddividere lo schermo in molteplici settori e la facilità di utilizzare i diversi strumenti di collaborazione, scritta e orale, da parte di tutti i partecipanti alle lezioni. È importante sottolineare, inoltre, che molte piattaforme telematiche stabiliscono limiti, anche significativi, sulla grandezza, sul formato e sull'estensione dei file condivisibili con la classe in tempo reale.

Le sessioni didattiche sincrone, analogamente alle lezioni in aula, si presentano a tutti i partecipanti nelle fattezze di un'esperienza multisensoriale, in cui le diverse capacità espressive (a cominciare dai 4 skills principali, ovvero ascolto, lettura e produzione orale e scritta in lingua straniera) possono essere stimolate ed attivate attraverso la rotazione e la diversificazione dei materiali multimediali utilizzati. Nel contesto dell'esposione di contenuti grammaticali 
congiuntamente all'uso di realia, ad esempio, lo strumento telematico si è rivelato un valido dispositivo per la condivisione di documenti e immagini in maniera rapida e capillare. Il fatto che il materiale sia reso accessibile, in tempo reale e senza perdite di tempo, direttamente sull'apparecchiatura utlizzata da ciascuno studente sembra tradursi, in particolare, in una articolata ed efficace stimolazione della partecipazione individuale.

Nelle fasi delle lezioni dedicate alla discussione e all'elicitazione collettiva delle strutture linguistiche e grammaticali prese in considerazione durante l'esposizione, è emersa per gli allievi l'importanza di potersi esprimere, a seconda dei diversi stili di apprendimento, in forma sia orale sia scritta e, in alcuni casi, anche simultaneamente, ad esempio digitando parole e frasi nella chat di classe e al contempo intervenendo a viva voce. Nella didattica sincrona a distanza, tuttavia, è emersa anche l'impossibilità da parte degli studenti di fornire, per motivi prettamente tecnici, risposte orali corali (di classe o di gruppo), contrariamente a quanto avviene in aula.

Nelle fasi sincrone dedicate all'applicazione pratica dei contenuti di ciascuna lezione, la presenza del docente e la possibilità di porre domande e richiedere chiarificazioni in tempo reale sono di importanza assoluta per la maggioranza degli studenti. Infine, per quanto riguarda la produzione aperta di contenuti nel corso delle sessioni sincrone, molti allievi criticano la lentezza ed i limiti delle piattaforme telematiche e delle loro modalità di organizzazione, suddivisione e gestione dei gruppi di lavoro a distanza. Dal momento che il docente può essere presente in un solo gruppo di lavoro alla volta, inoltre, contrariamente a quanto avviene in aula questi non sempre riesce a monitorare, anche per la relativa lentezza dei tempi tecnici di connessione, l'operato di tutti i partecipanti. 


\subsection{Componenti asiconrone}

Nella presentazione asincrona del materiale didattico è di fondamentale importanza ordinare le diverse tappe dell'apprendimento in maniera chiara, lineare e, quando possibile, intuitivamente comprensibile. Particolarmente utile si è rivelato, a questo proposito, l'uso di check-lists di completamento congiuntamente all'adozione di tabelle riassuntive indicanti i contenuti di ciascuna lezione e modulo didattico. A una percentuale signficativa di studenti risulta molto gradita un'accorta indicizzazione degli handouts e dei realia utilizzati, così come l'inclusione di organigrammi indicanti la collocazione del materiale oggetto di studio all'interno della più ampia suddivisione mensile e semestrale dei contenuti del corso.

In virtù della versatilità dello strumento telematico, ed anche ai fini di avvantaggiare i diversi stili cogntivi e di sollecitare codici diversificati di apprendimento (Torresan 2016), si è rivelato di grande utilità variare, in maniera programmatica e costante, il formato e la tipologia delle attività asincrone del corso. La possibilità di inserire links e collegamenti a materiale multimediale di varia natura (ad esempio riviste e giornali in linea, blog, siti internet, videoclip musicali, estratti da film e sceneggiati, documenti audiovisivi liberamente consultabili online, ecc.) è in generale percepita positivamente o molto positivamente dagli studenti, così come la possibilità di prendere in considerazione più volte, anche consecutivamente, un medesimo documento multimediale una volta che questo venga reso permanentemente disponibile sul sito del corso.

Particolarmente apprezzato dagli studenti è l'utilizzo di documenti reali, e attuali, di breve o di brevissima lunghezza. Presentati congiuntamente al materiale di studio, questi realia paiono dimostrarsi particolarmente efficaci nello stimolare l'attenzione degli allievi fornendo loro, oltre all'opportunità di esercitare in vario modo le proprie competenze linguistiche e cognitive, la 
possibilità di entrare a contatto con alcuni aspetti e tematiche appartenenti alla cultura italiana e italofona del passato e del presente.

Nei corsi di italiano per principianti del semestre autunnale 2020 a Dalhousie, a titolo di esempio, sono stati utilizzati tra l'altro: videoclip musicali di Carl Brave e Elodie (Parli parli, 2020, per la descrizione di persone e azioni), Fedez (Prima di ogni cosa, 2019, per aggettivi possessivi e termini di parentela) e Audio2 (Sono le venti, 1995, l'ora); brevi articoli e biografie di persone famose (chiecosa.it, per attività di lettura rapida e ricerca di informazioni); fotogrammi da film e sceneggiati (per la descrizione di luoghi e di persone); brevi estratti da registrazioni video di performance musicali (Vivaldi, Le quattro stagioni, per il vocabolario); realia provenienti da quotidiani e riviste in italiano (repubblica.it, vogue.it ed altri); altri documenti d'archivio, come sigle iniziali e titoli di telegiornali e brevi estratti da programmi televisivi tra cui, sempre a titolo di esempio, Che tempo fa con Edmondo Bernacca (Rai, 1979).

\section{Verifiche e valutazione}

Le peculiarità e le caratteristiche intrinseche all'insegnamento per via telematica richiedono, nelle fasi di progettazione e di preparazione dei corsi, un'attenta riflessione preventiva relativa alle modalità di valutazione del processo di apprendimento degli allievi e, congiuntamente, dell'operato del docente. In questa cornice, pratiche valutative di tipo formativo (da svilupparsi, ad esempio, su base settimanale) sono sicuramente preferibili alla somministrazione di verifiche puramente sommative, specialmente se presentate a larghi intervalli di tempo. La misurazione del progresso di ciascuno studente si è svolta, nel nostro caso, su base settimanale, tramite l'assegnazione di compiti (valutati con formula numerica, ovvero con veri e propri voti) comprendenti una varietà di brevi esercizi "allineati" (per 
utilizzare nuovamente il quadro teorico di Biggs 2003) ai contenuti del corso e, soprattutto, alle attività comunicative svolte in sede sincrona e asincrona.

L'utilizzo di una piattaforma telematica efficiente e versatile risulta essere un prerequisito essenziale anche per quanto riguarda la somministrazione di compiti e di verifiche formative, diversificate e multimediali, rispettose dei diversi stili di apprendimento e centrate sull'aquisizione di capacità comunicative contestualizzate ed effettuali. Il feedback degli studenti suggerisce in particolare che l'attivazione delle capacità di lettura, scrittura, ascolto e produzione orale in un contensto diversificato, soprattutto se connesso all'utilizzo di documenti reali e attuali, è preferibile agli esercizi mnemonici e alla produzione di contenuti (o alla mera applicazione di regole) sganciata o separata da un utilizzo realistico della lingua straniera oggetto di studio.

Anche in virtù delle preferenze espresse dagli allievi, i compiti settimanali sono stati suddivisi in un numero variabile di sezioni, diverse tra di loro in termini sia di contenuto che di modalità di completamento. Più specificatamente, sono stati proposti esercizi di ascolto e di lettura di brevi brani accompagnati da domande a risposta semplice, a risposta multipla e a risposta aperta; esercizi di produzione orale, spesso in forma di domande e dialoghi in prima e in seconda persona; esercizi di coniugazione e di utilizzo di strutture-chiave connesse ai contenuti delle sessioni sincrone e asincrone; produzione di brevi brani, scritti e orali, su temi legati all'esperienza personale di ciascun allievo oppure ai realia oggetto di studio.

L'opportunità e la possibilità di creare e condividere rapidamente documenti e materiali multimediali è percepita positivamente o molto positivamente dalla maggioranza degli studenti. Per oltre la metà degli intervistati, infatti, la possibilità di ricevere correzioni e suggerimenti personalizzati in forma non soltanto scritta ma anche orale (ad esempio 
tramite file audio e video) contribuisce in maniera sostanziale non solo all'apprendimento ma anche ad una positiva percezione dell'utilità del corso nel suo insieme. L'opportunità di poter creare, condividere e scambiare file in tempo reale corrisponde inoltre, per circa un terzo allievi, a un fattore qualificante dei corsi, in quanto permette di monitorare efficacemente il proprio apprendimento e di ricevere agevolmente correzioni e suggerimenti personalizzati. Per il docente, infine, la possibilità di fornire a ciascun allievo un feedback dettagliato e capillare, nonché rispettoso dei diversi stili di apprendimento, presenta il vantaggio di permettere la tempestiva circolazione di informazioni, suggerimenti e correzioni.

\section{Dati statistici}

Dalhousie è un'università pubblica il cui corpo studentesco consta di circa 15000 allievi, a cui si aggiungono circa 4500 studenti di master e di dottorato. L'università è sita nella città di Halifax, capoluogo della provincia canadese e angolofona della Nuova Scozia, e proprio da questa provincia proviene il 18\% circa degli studenti iscritti, nel 2020, ai corsi. Circa il 60\% degli studenti proviene dalle altre provincie del Canada; il rimanente $22 \%$ è di estrazione internazionale.

Ogni semestre accademico, il Programma di Italiano propone un minimo di due e un massimo di tre corsi di lingua italiana per principianti. Ciascun corso ha una capienza di 30 studenti. Nel decennio 2010-2020, i corsi di italiano sono sempre iniziati a capienza massima raggiunta. A distanza di tre settimane dal primo giorno di lezione, tuttavia, in tutti i semestri accademici antecedenti il 2020 si è sempre registrato un aumento di circa il 10\% nel numero di studenti iscritti alle lezioni di lingua italiana, principalmente a causa della promozione dei corsi di italiano spontaneamente effettuata, tramite 
passaparola, nel corso delle prime settimane di lezione da parte degli studenti già iscritti.

\subsection{Iscrizioni}

Nell'autunno 2020, a Dalhousie sono stati offerti tre corsi di italiano per principianti. All'inizio del semestre, a ciascun corso erano iscritti trenta studenti, per un totale complessivo corrispondente al raggiungimento della capienza massima in tutti e tre i corsi. Se messe a confronto con i dati raccolti nel decennio precedente, queste cifre sembrano indicare la neutralità, se non la sostanziale mancanza di effetti immediatamente tangibili, della transizione dalla didattica di persona a quella a distanza sul numero totale di iscritti ai corsi. A tre settimane dall'inizio delle lezioni, tuttavia, nell'autunno 2020 non si è registrato alcun aumento nel numero delle iscrizioni. Contrariamente a quanto avveniva in passato, il passaparola tra studenti non si è verificato e questo, congiuntamente alla mancata possibilità di presenziare ad una o più lezioni prima di decidere se iscriversi o meno ai corsi, ha influito negativamente sul numero totale di allievi causando una diminuzione netta di circa il 10\% degli effettivi.

\subsection{Abbandoni}

Nel corso di ogni semestre accademico si verificano, per una grande varietà di cause spesso connesse al vissuto personale di ciascuno studente, una serie di abbandoni. Il tasso semestrale di abbandono dei corsi di italiano, che per molti allievi coincide con l'abbandono dei corsi universitari tout court e che nel periodo 2010-20 ha interessato su base annuale circa lo 0,6 \% degli iscritti, è rimasto nell'autunno 2020 pressoché invariato. Un numero significativo di allievi, tuttavia, ha progressivamente smesso di partecipare alle attività di classe (sia nelle loro componenti sincrone, ovvero l'incontro plenario settimanale di 
classe, sia nelle componenti asincrone, ovvero la consultazione di materiali online) non giungendo a fine semestre al raggiungimento degli obiettivi minimi, linguistici e formativi, del corso. Di fronte ad un tasso di insuccesso a fine semestre attestatosi intorno ad un massimo dell'1,8\% negli anni accademici compresi tra il 2010 ed il 2020, nell'autunno 2020 il 6,3\% degli studenti non ha ricevuto crediti formativi per i corsi di italiano.

Le ragioni di tale significativo incremento vanno ricercate non tanto nelle pratiche didattiche adottate nei corsi di lingua italiana, quanto in una più generale perdita di interesse nei confronti dell'insegnamento universitario a distanza. A questo proposito è altamente indicativa, nelle risposte aperte degli allievi a quesiti relativi alla didattica online, la ricorrente presenza di parole chiave quali "malessere" e "disagio", spesso congiuntamente ad espliciti riferimenti alla difficoltà di organizzare in maniera "autonoma" le tempistiche ed il ritmo dell'apprendimento. Per un numero maggioritario di studenti, una delle cause principali del senso di "sfiducia" e di "disaffezione" sviluppatosi nei confronti degli studi universitari è da ricercarsi nell'impossibilità di incontrare "di persona" i docenti e gli altri allievi. Particolarmente significativo, a questo proposito, è l'uso da parte quasi un terzo degli intervistati di termini e concetti strettamente connessi al tema dell'interazione interpersonale quali "appartenenza", "comunità (community)", "dialogo" e "fattore umano".

Il sintomatico utilizzo, da parte di molti degli studenti intervistati, delle categorie interpretative sopraccitate non sembra essere connesso alle specificità, al contenuto né alle attività didattiche dei corsi di italiano. Un simile e più generale senso di "frustrazione" e di "insoddisfazione" emerge infatti, in percentuali anche drammatiche, dai sondaggi che sono stati effettuati a metà semestre, per conto della Facoltà di Arti e Scienze Sociali di cui il Programma 
di Italiano fa parte, relativi alla percezione, da parte del corpo studentesco, della didattica a distanza nel suo insieme.

\section{Conclusioni}

Dall'osservazione dei diversi parametri ed indicatori di successo della didattica a distanza nell'ambito dei corsi offerti dal Programma di Italiano dell'Università Dalhousie nell'autunno 2020, emergono risultati ambivalenti. Se, da un lato, l'insegnamento-apprendimento per via telematica presenta per il docente alcuni significativi vantaggi, quali l'opportunità di sviluppare un monitoraggio costante e individualizzato del progresso di ciascun allievo (anche in virtù della strumentazione tecnica, che permette di fornire un feedback preciso, accurato, personalizzato e multimediale) e la possibilità di includere nel materiale didattico realia e altri documenti multimediali difficilmente utilizzabili in aula, dall'altro lato l'insegnamento a distanza sembra privare il processo di insegnamento-apprendimento di alcune pratiche collaborative, sviluppabili esclusivamente di persona e dalla dimensione squisitamente umana, quali l'interazione di classe e il lavoro, in gruppi e a coppie, di persona.

L'insegnamento a distanza si traduce in un processo dalle valenze contradditorie anche nella percezione degli studenti. Come si evince dalle risposte ai questionari periodicamente somministrati agli iscritti ai corsi, infatti, l'insegnamento per via telematica presenta alcuni significativi punti di forza, come la possibilità per gli allievi di avvicinare il materiale didattico asincrono in qualsiasi momento della giornata e della settimana, ma anche notevoli limiti, come la mancanza di una routine stabile e costante che aiuti ad organizzare in maniera accorta e bilanciata il ritmo dello studio giornaliero e settimanale. Le difficoltà legate all'organizzazione in proprio delle tempistiche di apprendimento si traducono, per una percentuale significativa di allievi, in un 
elevato tasso di abbandoni. Così come avviene per l'insegnamento di persona, il costante monitoraggio del feedback degli iscritti ai corsi costituisce un efficace strumento diagnostico e fornisce un potenziale antidoto all'abbandono degli studi universitari da parte di una percentuale minoritaria, ma non per questo meno importante, di allievi.

\section{Riferimenti}

BIGGS, John. "Enhancing Teaching Through Constructive Alignment", Higher Education, 32, 347-364, 1996.

. "Aligning Teaching for Constructing Learning", The Higher Education Academy Resources. Disponibile su: < https://www.advancehe.ac.uk/knowledge-hub/aligning-teaching-constructing-learning $>$. Data dell'ultimo accesso: 10 dicembre 2020.

. "What the Student Does: Teaching for Enhanced Learning", Higher Education Research \& Development, 31 (1), 39-55, 2012.

BLACK, Paul and WILIAM, Dylan. "Inside the Black Box: Raising Standards Through Classroom Assessment”, Phi Delta Kappan, 80 (2), 139-148, 1998.

BRUSCHI, Isabella e MATTEUCCI, Paolo. "Tutor e tirocinante condividono un percorso di Ricerca-Azione”, Lingua e Nuova Didattica, 32 (4), 96-106, 2003.

GIBBS, Graham and SIMPSON, Claire. "Conditions under Which Assessment Supports Students Learning", Learning and Teaching in Higher Education, 1 (1), 331, 2004.

HARRIS, Marvin. Cultural Anthropology. New York: Harper \& Row, 1983.

LOSITO, Bruno e POZZO, Graziella. La ricerca azione. Una strategia per il cambiamento nella scuola. Roma: Carocci, 2005.

POZZO, Graziella e RIZZARDI, Maria Cecilia. "La ricerca azione: una strategia per il cambiamento", Lingua e Nuova Didattica, 32 (4), 7-24, 2003.

POZZO, Graziella e ZAPPI, Liliana. Ricerca-azione. Metodiche, strumenti, casi. Torino: Bollati Boringhieri, 1993. 
RAMSDEN, Paul. "A Performance Indicator of Teaching Quality in Higher Education: The Course Experience Questionnaire", Studies in Higher Education, 16 (2), 129-150, 1991.

TORRESAN, Paolo. "Didattica dell'italiano, intelligenze, risorse digitali", Revista Italiano UERJ, 7 (1), 81-94, 2016.

TRIPP, David. "Action-research: a methodological introduction", Educaşão e Pesquisa, 31 (3), 443-466, 2005.

WILIAM, Dylan. "What is Assessment for Learning?", Studies in Educational Evaluation, 37 (1), 3-14, 2011. 